\title{
Jasminanthes xuanlienensis (Apocynaceae, Asclepiadoideae), a new species from Vietnam
}

\author{
The Bach Tran', Do Van Hai', Bui Thu $\mathrm{Ha}^{2}$, Michele Rodda ${ }^{3}$ \\ I Department of Botany, Institute of Ecology and Biological Resources, Vietnam Academy of Science and \\ Technology, 18 Hoang Quoc Viet, Cau Giay, Hanoi, Vietnam 2 Hanoi National University of Education, \\ 136, Xuan Thuy Street-Cau Giay District, Hanoi, Vietnam 3 The Herbarium, Singapore Botanic Gardens, 1 \\ Cluny Road, 259569 Singapore
}

Corresponding author: Michele Rodda (rodda.michele@gmail.com)

Academic editor: C. Morden | Received 18 May 2016 | Accepted 30 July 2016 | Published 18 August 2016

Citation: Tran TB, Hai DV, Ha BT, Rodda M (2016) Jasminanthes xuanlienensis (Apocynaceae, Asclepiadoideae), a new species from Vietnam. PhytoKeys 69: 17-22. doi: 10.3897/phytokeys.69.9272

\begin{abstract}
Jasminanthes xuanlienensis (Apocynaceae, Asclepiadoideae), a new species from Vietnam is described, illustrated and compared with its five congeners. Jasminanthes xuanlienensis differs distinctly from congeners by the longer peduncles (14-18 cm vs. $4 \mathrm{~cm}$ at most in J. pilosa and J. saxatilis, salmon-pink color of the inner corolla lobes (white or greenish in the other species), and corolla tube length (12.0-14.5 mm vs. shorter or longer in congeners).
\end{abstract}

\section{Keywords}

Marsdenieae, China, Xuan Lien National Park

\section{Introduction}

Jasminanthes Blume (Apocynaceae, Asclepiadoideae, Marsdenieae) is a small Old World genus of six species (Endress et al. 2014). Its type species, Jasminanthes suaveolens Blume, is from Java. However, the centre of diversity of Jasminanthes is mainland Asia, particularly China, where the remaining congeners are found (Gilbert et al. 1995, $\mathrm{Li}$ et al. 1995). Jasminanthes was considered to be a large-flowered Marsdenia R.Br. by Forster (1995). However, the very broad circumscription of Marsdenia adopted by Forster is not based on phylogenetic evidence and has not been generally accepted 
in later works. For instance Gymnema, also considered congeneric with Marsdenia by Forster (1995), was retained in floristic works such as Jagtap and Singh (1999) and Watson (1999). The phylogeny of Surveswaran et al. (2014) does not address whether Gymnema should be merged with Marsdenia. The purpose of the present paper is limited to validate a new taxon and we believe that its correct placement is in Jasminanthes. Jasminanthes is separated from Marsdenia based on its large flowers $(>15 \mathrm{~mm}$ long) with salverform corolla and by its inconspicuous to absent staminal corona. In contrast, Marsdenia usually has much smaller flowers ( $<10 \mathrm{~mm}$ long) with urceolate or rotate corolla and conspicuous staminal corona. If molecular evidence later proves that Jasminanthes is indeed to be considered within a broadly circumscribed Marsdenia, a new combination will be required.

In Vietnam, no species of Jasminanthes have been recorded to date (Ho 1993, Tran 2005). However, recent fieldwork in Xuan Lien National Park, Vietnam, yielded a collection of Jasminanthes that was identified as a new species based upon comparison with the known species in the literature and specimens at BK, BKF, BM, HN, HNU, HNPM, IBK, IBSC, K, KUN, KYO, P, SING, TI, TO, TUT, and VNM. Here we describe the new species and provide a detailed table of character differences among the species along with a key to the species of Jasminanthes.

\section{Species treatment}

Jasminanthes xuanlienensis T.B.Tran \& Rodda, sp. nov. urn:Isid:ipni.org:names:60472843-2

Fig. 1

Type. Vietnam. Thanh Hoa province: Xuan Lien National Park, 720 m, N1959'14.6", E104'59'49.1", 22 April 2013, Do Van Hai et al. XL 904 (HN, holotype; HN, isotype).

Description. Liana large, up to $10 \mathrm{~m}$ long. Stems glabrous, $1.7-2 \mathrm{~mm}$ in diameter; internodes $21-23 \mathrm{~cm}$ long. Leaves opposite; petiole $1.6-2.6 \mathrm{~cm}$ long, $1.5-2 \mathrm{~mm}$ in diameter, pubescent with spreading trichomes, lamina variable in shape, elliptic (ovate), $11-15 \times 4.1-6.8 \mathrm{~cm}$, with many black spots when dry; adaxial surface glabrous except sparsely pubescent base with spreading trichomes, basal colleters $1-3$ each lamina base, ovoid; abaxial surface pubescent, with spreading trichomes; base round to acuminate; apex acuminate with a caudate tip $0.8-1.5 \mathrm{~cm}$ long; lateral veins 9-13 pairs. Inflorescences extra-axillary, simple or dichotomous, umbelliform, up to 30-flowered; peduncle 14-18 cm long, $1.3-1.7 \mathrm{~mm}$ in diameter, pubescent, trichomes spreading to retrorse. Pedicel $8.5-11.0 \mathrm{~mm}$ long, $0.2-0.5 \mathrm{~mm}$ in diameter, pubescent with spreading trichomes. Flower bud just before anthesis fusiform, 10.7-13.0 mm long, 1.9-2.9 mm in diameter, apex acuminate, base bulbous. Calyx sepals 5, free; lobes triangular-linear, apex acuminate, $2.5-2.6 \times 0.6-1.0 \mathrm{~mm}$, adaxial surface glabrous, abaxial surface pubescent with spreading trichomes; colleters occurring singly between the sepals, ovoidconical, $0.2-0.3 \times 0.1-0.2 \mathrm{~mm}$. Corolla salverform, tube $12.0-14.5 \mathrm{~mm}$ long, adaxi- 

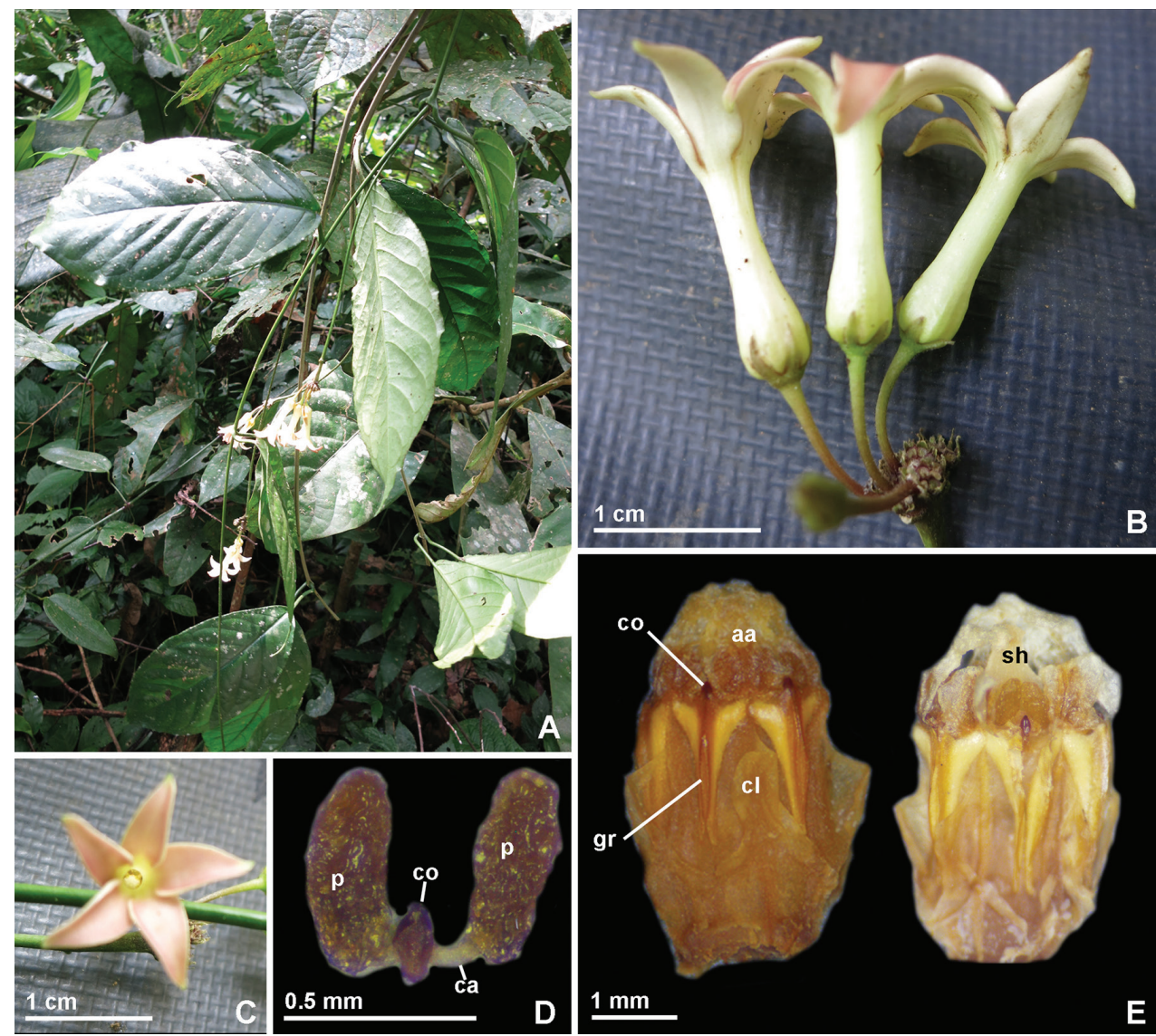

Figure I. Jasminanthes xuanlienensis T.B.Tran \& Rodda. A Flowering branch in the habitat of the type locality (22 April 2013) B Inflorescence C Flower, top view D Pollinarium E Gynostegium and staminal corona. aa: anther appendage; ca: caudicle; cl: corona lobe; co: corpusculum; gr: guide rail; p: pollinium; sh: style head . (Photographs by T.B. Tran, photo edit by M. Rodda).

ally with 5 pairs of longitudinal lines of retrorse trichomes, abaxially glabrous, throat pubescent, eglandular trichomes $0.36-0.70(-1.34) \mathrm{mm}$ long, lobes elliptic-ovate to triangular, 7.0-8.0 × 3.0-3.6 mm, adaxial surface salmon-pink, abaxial surface greenish-white. Corona staminal; corona lobes arrow-shaped, much reduced and attached to gynostegium lengthwise, c. $1.8 \mathrm{~mm}$ high, c. $0.8 \mathrm{~mm}$ wide, glabrous. Guide rails $1.7-2.0 \mathrm{~mm}$ long. Gynostegium $3.5-4.0 \mathrm{~mm}$ tall, $1.8-2.0 \mathrm{~mm}$ wide. Pollinia erect, oblong, $0.49-0.58 \mathrm{~mm} \times 0.2-0.23 \mathrm{~mm}$; caudicle $0.12-0.13 \mathrm{~mm} \times 0.05-0.06 \mathrm{~mm}$; corpusculum rhomboid, $0.2-0.23 \times 0.10-0.11 \mathrm{~mm}$. Anther appendages erect, covering the style head, $1.0-1.2 \times 0.7-0.9 \mathrm{~mm}$. Style head conical with a round tip, $0.8-1.0 \mathrm{~mm}$ high. Ovary bi-carpellate, carpels ovoid, c. $1.60 \mathrm{~mm}$ long, $1.10 \mathrm{~mm}$ wide at the base. Fruits and seeds not observed.

Etymology. The species is named after the type locality, Xuan Lien National Park, Thanh Hoa province, in northern Vietnam. 
Table I. Morphological comparision of Jasminanthes species.

\begin{tabular}{|c|c|c|c|c|c|c|}
\hline Characters & J. xuanlienensis & J. chunii & J. mucronata & J.pilosa & J. saxatilis & J. suaveolens \\
\hline Lamina shape & elliptic (ovate) & ovate & ovate-oblong & $\begin{array}{l}\begin{array}{l}\text { oblong- } \\
\text { ovate }\end{array} \\
\end{array}$ & $\begin{array}{c}\text { elliptic- } \\
\text { lanceolate }\end{array}$ & $\begin{array}{l}\text { elliptic- } \\
\text { lanceolate }\end{array}$ \\
\hline Lamina base & acuminate & $\begin{array}{c}\text { cordate- } \\
\text { subcordate }\end{array}$ & $\begin{array}{l}\text { cordate- } \\
\text { subcordate }\end{array}$ & cordate & acute & acute to round \\
\hline Lamina apex & caudate & $\begin{array}{c}\text { acute- } \\
\text { cuspidate }\end{array}$ & $\begin{array}{l}\text { acuminate- } \\
\text { short caudate }\end{array}$ & $\begin{array}{c}\text { shortly } \\
\text { acuminate }\end{array}$ & $\begin{array}{c}\text { acuminate- } \\
\text { caudate }\end{array}$ & $\begin{array}{c}\text { acuminate- } \\
\text { cuspidate }\end{array}$ \\
\hline $\begin{array}{l}\text { Lateral veins } \\
\text { (pairs) }\end{array}$ & $9-13$ & $4-7$ & $5-7$ & $7-10$ & $5-6$ & 5-8 \\
\hline $\begin{array}{l}\text { Length of } \\
\text { petiole }(\mathrm{cm})\end{array}$ & $1.6-2.6$ & $1-1.5(-2)$ & $1.5-3$ & $1-4.5$ & $1.3-1.8$ & $0.7-1.2$ \\
\hline $\begin{array}{l}\text { Number of } \\
\text { flowers }\end{array}$ & up to 30 & up to 12 & $2-4(-9)$ & $5-10$ & c. 10 & c. 10 \\
\hline $\begin{array}{l}\text { Length of } \\
\text { peduncle }(\mathrm{cm})\end{array}$ & $14-18$ & $\begin{array}{l}(0.5-) 1- \\
1.5 \mathrm{~cm}\end{array}$ & $1-2$ & $2-4 \mathrm{~cm}$ & $3-4$ & $0.5-1$ \\
\hline $\begin{array}{l}\text { Length of } \\
\text { pedicel }(\mathrm{cm})\end{array}$ & $0.85-1.1$ & $1-1.3$ & $1-3$ & $0.7-1$ & $0.8-1$ & $0.7-1$ \\
\hline Shape of sepal & triangular & oblong & elliptic-oblong & $\begin{array}{c}\text { oblong- } \\
\text { lanceolate }\end{array}$ & triangular & triangular \\
\hline $\begin{array}{l}\text { Size of sepal } \\
(\mathrm{mm})\end{array}$ & $2.5-2.6 \times 0.6-1$ & $5-6 \times$ c. 2 & $7-8 \times 3-4$ & $\begin{array}{l}18-30 \times \\
4-8 \mathrm{~mm} \\
\end{array}$ & c. $3 \times 1$ & $5.5-8 \times 2-3.5$ \\
\hline $\begin{array}{l}\text { Corolla lobe } \\
\text { colour }\end{array}$ & $\begin{array}{l}\text { adaxially salmon- } \\
\text { pink, abaxially } \\
\text { greenish-white }\end{array}$ & white & white & white & greenish & white \\
\hline $\begin{array}{l}\begin{array}{l}\text { Length of } \\
\text { corolla tube } \\
(\mathrm{mm})\end{array} \\
\end{array}$ & $12.0-14.5$ & 7-9 & c. 15 & $40-50$ & $6-8$ & $9-11$ \\
\hline $\begin{array}{l}\text { Size of corolla } \\
\text { lobe }(\mathrm{mm})\end{array}$ & $\begin{array}{c}7.0-8.0 \times \\
3.0-3.6\end{array}$ & $6-8 \times 4-5$ & $15-17 \times 6-8$ & $\begin{array}{c}25-30 \times \\
6-8\end{array}$ & c. $10 \times 3$ & $\begin{array}{c}7.0-9.0 \times \\
1.0-1.5\end{array}$ \\
\hline
\end{tabular}

Distribution and ecology. Jasminanthes xuanlienensis was found growing in primary evergreen forests on limestone soil of Xuan Lien National Park. It was collected in flower in April. Plants observed growing in the vicinity include Piper acreanum C.DC., Beccarinda tonkinensis (Pellegr.) B.L.Burtt, Sarcosperma kachinense (King \& Prain) Exell, Hoya vercillata (Vahl) G.Don, and Alangium salviifolium (L.f.) Wangerin.

Conservation status. Jasminanthes xuanlienensis is known only from the type locality, an area still poorly known botanically; its preliminary conservation status is therefore Data Deficient (DD; IUCN 2014).

Notes. The six species of Jasminanthes are clearly distinguishable from one another based on morphological characters (Table 1). The new species can be easily separated from all Jasminanthes species as it has very long peduncles (14-18 cm long), while the longest peduncles observed in other species are those of $J$. pilosa (Kerr) W.D.Stevens \& P.T.Li and J. saxatilis (Tsiang \& P.T.Li) W.D.Stevens \& P.T.Li), which reach 4 $\mathrm{cm}$ long. Jasminanthes xuanlienensis has unique pink corolla lobes whereas the other species have white corollas, or greenish corollas in J. saxatilis. 


\section{Key to the species of Jasminanthes}

1 Peduncle $>10 \mathrm{~cm}$ long; corolla salmon, pink adaxially .........J. xuanlienensis

- $\quad$ Peduncle $<5 \mathrm{~cm}$ long; corolla white or greenish adaxially ...........................2

2 Sepals $>15 \mathrm{~mm}$ long; corolla tube $>35 \mathrm{~mm}$ long............................... J. pilosa

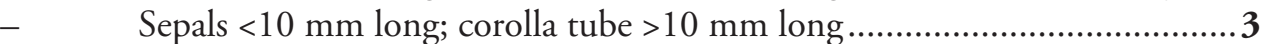

3 Corolla tube $>13 \mathrm{~mm}$ long, lobes $>14 \mathrm{~mm}$ long ........................ mucronata

- $\quad$ Corolla tube $<11 \mathrm{~mm}$ long, corolla lobes $<10 \mathrm{~mm}$ long .............................4

$4 \quad$ Lamina ovate, base cordate or subcordate ....................................... J. chunii

- Lamina elliptic-lanceolate, base acute to round ......................................... 5

$5 \quad$ Sepals $<3.5 \mathrm{~mm}$ long; peduncle $>3 \mathrm{~cm}$ long................................ J. saxatilis

- Sepals $>5 \mathrm{~mm}$ long; peduncle $<1.5 \mathrm{~cm}$ long................................ suaveolens

\section{Acknowledgments}

We thank the directors and curators of BK, BKF, BM, HN, HNU, HNPM, IBK, IBSC, K, KUN, KYO, P, SING, TI, TO, TUT and VNM herbaria for allowing access and/or for providing high quality images of herbarium specimens. The scientists of the Department of Botany, Institute of Ecology and Biological Resources (Hanoi) are thanked for collecting specimens. The National Parks Board Singapore is thanked for supporting this research by sponsoring numerous herbaria study trips. Lastly, we thank two anonymous reviewers and editor Clifford Morden for their contributions to the text. Funding for this research was provided by NAFOSTED project "Bioprospecting on Biological Materials of Vietnam".

\section{References}

Endress ME, Liede-Schumann S, Meve U (2014) An updated classification for Apocynaceae. Phytotaxa 159: 175-194. doi: 10.11646/phytotaxa.159.3.2

Forster PI (1995) New names and combinations in Marsdenia (Asclepiadaceae: Marsdenieae) from Asia and Malesia (excluding Papuasia). Australian Systematic Botany 8: 691-701. doi: 10.1071/SB9950691

Gilbert MG, Stevens WD, Li PT (1995) Notes on the Asclepiadaceae of China. Novon 5: 1-16. doi: $10.2307 / 3391820$

Ho PH (1993) Cay co Viet Nam: An illustrated flora of Vietnam. Mekong Printing, Montreal. Jagtap AP, Singh NP (1999) Fascicle 24 (Apocynaceae, Asclepiadaceae). Fascicles of Flora of India, 24. Botanical Survey of India, Calcutta.

IUCN Standards and Petitions Subcommittee (2014) Guidelines for using the IUCN Red List categories and criteria. Version 11. Prepared by the Standards and Petitions Subcommittee. Downloadable from http://www.iucnredlist.org/documents/RedListGuidelines.pdf 
Li PT, Gilbert, MG, Stevens WD (1995) Asclepiadaceae. In: Wu ZY, Raven PH (Eds) Flora of China, vol. 16. Sci. Press, Beijing \& Missouri Bot. Garden Press, St. Louis, 189-270.

Surveswaran S, Sun M, Grimm GW, Liede-Schumann S (2014) On the systematic position of some Asian enigmatic genera of Asclepiadoideae (Apocynaceae). Botanical Journal of the Linnean Society 174: 601-619. doi: 10.1111/boj.12156

Tran TB (2005) Asclepiadaceae. In: Ban NT (Ed.) Checklist of plant species of Vietnam 3. Agricultural Publishing House, Hanoi, Vietnam, 58-75.

Watson MF (1999) Asclepiadaceae. In: Long DG (Ed.) Flora of Bhutan 2: 687-733. 